\title{
The phytopathogenic bacteria Erwinia carotovora infects Drosophila and activates an immune response
}

\author{
Alan Basset*, Ranjiv S. Khush*, Anne Braun ${ }^{\dagger \neq}$, Louis Gardan§, Frédéric Boccard*, Jules A. Hoffmann ${ }^{\dagger}$, \\ and Bruno Lemaitre*ף \\ *Centre de Génétique Moléculaire, Centre National de la Recherche Scientifique, F-91198 Gif-sur-Yvette Cedex, France; ${ }^{\dagger}$ Institut de Biologie Moléculaire et \\ Cellulaire, Unité Propre de Recherche, 9022 du Centre National de la Recherche Scientifique, 15 Rue René Descartes, F-67084 Strasbourg Cedex, France; \\ and §Institut National de la Recherche Agronomique, Station de Phytobactériologie, Rue Georges Morel, 49070 Beaucouzé, France
}

Edited by Fotis C. Kafatos, European Molecular Biology Laboratory, Heidelberg, Germany, and approved January 28,2000 (received for review August 20, 1999)

\begin{abstract}
Although Drosophila possesses potent immune responses, little is known about the microbial pathogens that infect Drosophila. We have identified members of the bacterial genus Erwinia that induce the systemic expression of genes encoding antimicrobial peptides in Drosophila larvae after ingestion. These Erwinia strains are phytopathogens and use flies as vectors; our data suggest that these strains have also evolved mechanisms for exploiting their insect vectors as hosts. Erwinia infections induce an antimicrobial response in Drosophila larvae with a preferential expression of antibacterial versus antifungal peptide-encoding genes. Antibacterial peptide gene expression after Erwinia infection is reduced in two Drosophila mutants that have reduced numbers of hemocytes, suggesting that blood cells play a role in regulating Drosophila antimicrobial responses and also illustrating that this DrosophilaErwinia interaction provides a powerful model for dissecting host-pathogen relationships.
\end{abstract}

$\mathrm{n}$ response to microbial infection, Drosophila expresses a battery of genes that encode antibacterial (e.g., diptericin, cecropin, and attacin) and antifungal (e.g., drosomycin) peptides in the fat body. This immune response is controlled by signaling pathways that share similarities with the pathways that regulate innate immune responses in mammals $(1,2)$. A striking feature of the Drosophila immune response is its ability to differentiate between various classes of microorganisms and, at least in the case of fungi, to activate selectively the expression of specific genes encoding antifungal peptides (3). Fungi that infect Drosophila in the absence of septic injury have been identified (3); however, a bacterial species that induces the Drosophila immune response via natural infection has not been described. To date, studies of Drosophila defense against bacterial infection have relied on the introduction of bacteria directly into the body cavity by pricking or injection. To identify bacterial pathogens of Drosophila and to determine how Drosophila antibacterial responses are naturally activated and regulated, we screened for bacterial strains that activate Drosophila immune responses in the absence of physical injury.

Using this strategy, we identified members of the bacterial genus Erwinia that induce the systemic expression of antimicrobial peptide genes in Drosophila larvae after ingestion. The Erwinia genus is a member of the Gram-negative Enterobacteriaceae family, and various species are phytopathogenic, causing soft rots of fleshy fruits, vegetables, and ornamentals $(4,5)$. These pathogens have developed sustained plant-to-plant infection cycles, often via insect vectors such as bees and flies $(4,5)$. Drosophila melanogaster is a natural vector for Erwinia carotovora atroseptica and Erwinia carotovora carotovora, which cause potato blackleg disease. Drosophila carrying these species are found in potato fields, and at least under greenhouse conditions, Drosophila can spread blackleg disease among potato plants (6, 7). We speculate that the activation of Drosophila immune responses by some strains of Erwinia indicates that these bacteria exploit Drosophila as a host as well as a vector.

A central question in the regulation of Drosophila immune responses to microbial infection is the mechanism used to recognize invading microbes and subsequently to activate the expression of antimicrobial peptide genes in the fat body. By isolating a bacterial species that triggers the expression of these genes via a natural infection process, we now have a useful tool to address this question. Circulating blood cells (hemocytes) have been shown to have important roles in antibacterial defense, presumably by phagocytosing invading microbes (8). Although hemocytes can also produce antimicrobial peptides (1), recent data derived from bacterial injection experiments suggest that they are not required to activate the systemic antimicrobial response in the fat body (8). We examined antimicrobial peptide gene expression in Drosophila strains carrying the mutations domino (dom) and l(3) hematopoietic organ missing $(l(3)$ hem $)$, which reduce hemocyte numbers $(8,9)$. Antimicrobial peptide genes were induced in both of these mutants after bacterial injection; however, after natural Erwinia infection, the expression of the antibacterial peptide gene diptericin was reduced significantly. We present evidence that blood cells play a role in regulating the systemic antimicrobial response in Drosophila and illustrate the utility of this system for studying the regulation of both immune responses and host-pathogen interactions.

\section{Materials and Methods}

Drosophila Stocks. Oregon ${ }^{\mathrm{R}}\left(\mathrm{Or}^{\mathrm{R}}\right)$ flies were used as a standard wild-type strain. The transgenic strains diptericin-lac $Z$ (diptlacZ), drosomycin-lacZ (drom-lacZ), and cecropin-lacZ (ceclac $Z$ ) have been described (10-12). immune deficiency (imd) is a recessive mutation that strongly reduces the induction of all of the genes encoding antibacterial peptides after septic injury, while marginally affecting the expression of the antifungal peptide gene drosomycin (13). dom and l(3)hem mutations have been described elsewhere $(8,9)$. Drosophila stocks were maintained at $25^{\circ} \mathrm{C}$. Infected larvae were incubated at $29^{\circ} \mathrm{C}$.

Bacterial Strains. Bacteria were cultured in LB medium. The origin of the bacterial strains is described in the legend of

This paper was submitted directly (Track II) to the PNAS office.

Abbreviation: GFP, green fluorescent protein.

‡Present address: Massachusetts Institute of Technology, Biology Department, 77 Massachusetts Avenue, Cambridge, MA 02139

ITo whom reprint requests should be addressed. E-mail: lemaitre@cgm.cnrs-gif.fr.

The publication costs of this article were defrayed in part by page charge payment. This article must therefore be hereby marked "advertisement" in accordance with 18 U.S.C. $\S 1734$ solely to indicate this fact.

Article published online before print: Proc. Natl. Acad. Sci. USA, 10.1073/pnas. 070357597. Article and publication date are at www.pnas.org/cgi/doi/10.1073/pnas.070357597 


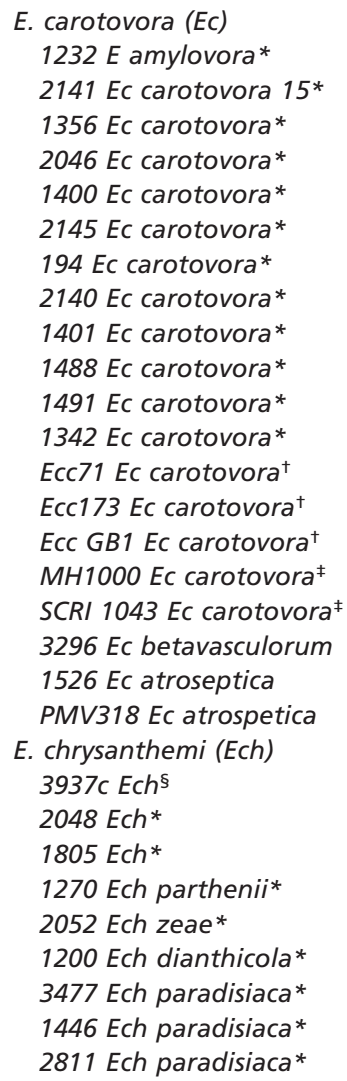

$$
\begin{gathered}
+ \\
++ \\
- \\
- \\
+ \\
+ \\
++ \\
++ \\
++ \\
++ \\
+ \\
+ \\
- \\
+ \\
++ \\
- \\
- \\
- \\
- \\
- \\
\\
- \\
- \\
+ \\
- \\
- \\
- \\
++ \\
+++ \\
+++
\end{gathered}
$$

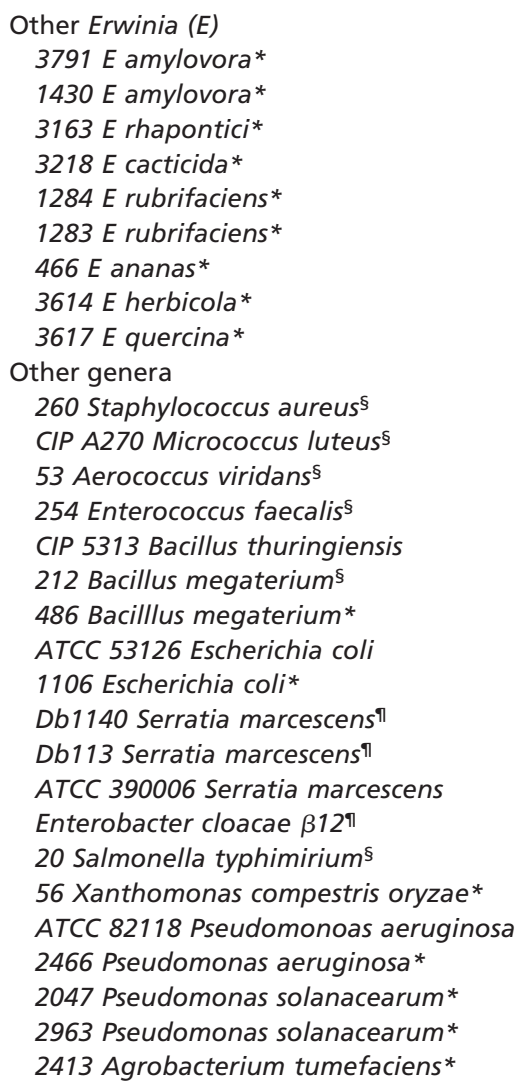

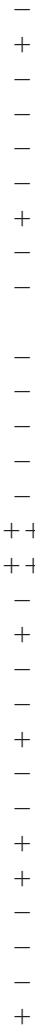

$\beta$-Galactosidase activity was assayed as previously described (3) in larvae carrying a dipt-lac Z reporter gene $24 \mathrm{~h}$ after natural infection. At least three independent measurements were performed on five larvae for each bacterial strain. - , None or very weak levels of $\beta$-galactosidase activity (mean $<2) ;+$, weak level of $\beta$-galactosidase activity $(10>$ mean $>2) ;++$, intermediate level of $\beta$-galactosidase activity $(40>$ mean $>10) ;+++$, strong level of $\beta$-galactosidase activity (mean $>$ 40). Bacterial strains were obtained from the following sources:

*Collection Française de Bacteries Phytopathogénes Center, Institut National de la Recherche Agronomique (Angers, France).

${ }^{\dagger}$ A. Chatterjee (Colombia, MO).

${ }^{\ddagger} \mathrm{G}$. Salmond, (Cambridge, U.K.).

sOur lab collection.

"H. G. Boman (Stockholm; ref. 25).

Table 1. Ecc-15 (2141) and Ecc-2046 (2046) were isolated on infected potato plants from France in 1978 and from Denmark in 1952, respectively. We isolated rifampicin-resistant mutants of Ecc-15 and Ecc-2046 (Ecc-15 rif $^{\mathrm{R}}$ and Ecc-2046rif ${ }^{\mathrm{R}}$ ) by using standard procedures (14). The green fluorescent protein (GFP)-expressing strain of Ecc-15 was generated by introducing plasmid pFPV25.1, which carries the GFP gene under the control of the Salmonella typhimurium rpSM promoter (15), via electroporation.

Infection Experiments. Bacterial injection. Third instar larvae were pricked with a thin needle inoculated with a concentrated bacterial pellet $\left(O D_{\approx 200}=4 \times 10^{11}\right.$ cells per $\left.\mathrm{ml}\right)$ from an overnight culture.

Natural bacterial infection. Approximately 200 third instar larvae were placed in a $2-\mathrm{ml}$ microfuge tube containing $200 \mu \mathrm{l}$ of concentrated bacterial pellet $\left(O D_{\approx 200}\right)$ from an overnight culture and $400 \mu \mathrm{l}$ of crushed banana. The larvae, bacteria, and banana were thoroughly mixed in the microfuge tube. The tube was stoppered with a foam plug and incubated at room temperature for $30 \mathrm{~min}$. The mixture then was transferred to a standard corn-meal fly medium and incubated at $29^{\circ} \mathrm{C}$. When Ecc- 15 was used in this infection procedure, $90 \%$ of the treated larva expressed genes encoding antimicrobial peptides in fat body cells; lower concentrations of Ecc-15 reduced the percentage of larvae that expressed these genes. Larvae were collected at different time intervals after infection for $\beta$-galactosidase assays (3), Northern blot analysis, and bacterial counts. For bacterial counting experiments, larvae and pupae were first rinsed in water, dipped in $70 \%(\mathrm{vol} / \mathrm{vol})$ ethanol $(3 \times 5 \mathrm{~s})$ for external sterilization, and then homogenized and spread onto LB plates.

Microscopic Observations. Live larvae infected by Ecc-15-GFP bacteria were anesthetized on ice and viewed under epifluorescent illumination (excitation filter $480 / 40 \mathrm{~nm}$; dichroic filter 505 $\mathrm{nm}$; emission filter $510 \mathrm{~nm}$ ) with a Leica (Heerburg, Switzerland) MZFLIII dissecting microscope. Images were recorded with a charge-coupled device camera (Sony). For hemolymph observations, larvae were sterilized in $70 \%$ (vol/vol) ethanol $(3 \times 5 \mathrm{~s})$ and then pricked to collect hemolymph. The hemolymph was observed directly under a microscope (Leitz DMRB) with either Nomarski optics or epifluorescent illumination.

Northern Blot Analysis. Total RNA extraction and Northern blotting experiments were performed as described in ref. 11. The following probes were used: attacin cDNA (16), cecropin A1 
cDNA (17), diptericin cDNA (10), drosomycin cDNA (18), and $r p 49$ cDNA (a PCR fragment of $\approx 400$ bp generated by using two oligonucleotides that were designed to amplify coding sequences of the ribosomal protein gene rp49; ref. 19).

\section{Results and Discussion}

Erwinia Strains Naturally Infect Drosophila Larvae. To identify a bacterial species that infects Drosophila naturally in the absence of physical injury, we focused on bacterial species that are found in wild Drosophila environments. D. melanogaster feed on yeasts associated with decaying fruits and vegetables, which also provide food and a habitat for developing larvae. This environment is host to various bacterial species, and previous studies show that D. melanogaster serves as a vector for at least a few, such as the phytopathogenic species of the bacterial genus Erwinia, that are associated with rotting plant matter $(6,7)$. We reasoned therefore that some Erwinia strains may also infect Drosophila and consequently activate immune responses. To screen for infection, we incubated Drosophila larvae carrying a dipt-lacZ reporter gene (10) in a mixture of crushed banana and bacteria and, $24 \mathrm{~h}$ later, assayed for the induction of the antibacterial peptide gene by measuring $\beta$-galactosidase activity levels.

We found that strains from two Erwinia species, Erwinia chrysanthemi paradisiaca and E. carotovora carotovora, are capable of triggering reproducible dipt-lacZ expression in Drosophila larvae (Table 1). All three isolates of E. chrysanthemi paradisiaca tested induce dipt-lac $Z$ expression; this bacterial species causes soft rot disease of banana trees, a common habitat for Drosophila in tropical areas, and we speculate that constant interactions between E. chrysanthemi paradisiaca and Drosophila have led to the exploitation of Drosophila as both a vector and a host. The different strains of E. carotovora carotovora tested differ strongly in their induction of dipt-lacZ expression, indicating that there is a high degree of specificity in their interactions with Drosophila. The infectious Erwinia strains do not, however, have a significant effect on larval viability, indicating that Drosophila defense mechanisms are capable of attenuating the infection (data not shown). We also tested species from other bacterial genera for their ability to infect Drosophila; of these additional strains, two isolates of Bacillus megaterium induced a weak but consistent expression of dipt-lacZ (Table 1). Finally, none of the additional Gram-negative or Gram-positive bacterial species that we tested induced dipt-lac $Z$ expression in larvae at consistent levels, suggesting that the relationship between Drosophila and the infectious Erwinia strains is the result of specific evolutionary adaptations in these bacteria that allow them to infect Drosophila (Table 1).

Natural Infection by Ecc-15 Induces a Global Immune Response. For our subsequent studies of Erwinia-Drosophila interactions, we selected two isolates of E. carotovora carotovora: isolate Ecc-15, which induces a strong immune response in feeding larvae as measured by $\beta$-galactosidase activity, and isolate Ecc-2046, which induces a weak response (Fig. $1 A$ ). The levels of dipt-lac $Z$ expression induced by natural Ecc-15 infection are only slightly lower than the levels induced by directly injecting Ecc- 15 into the larval body cavity, indicating that Ecc-15 is recognized efficiently by the larval defense system during a natural infection (Fig. $1 A$ and $C)$. Ecc- 15 cells killed by heat $\left(70^{\circ} \mathrm{C}\right.$ for $\left.40 \mathrm{~min}\right)$ induce a very weak larval immune response, suggesting that Ecc-15 must be viable to activate a full response, although it is possible that the induction is mediated solely by a bacterial cell surface or secreted factor that is inactivated by heat (Fig. $1 A$ ). Using Northern blot analysis, we monitored the kinetics of diptericin gene expression in larvae, pupae, and adults derived from third instar larvae that were infected by Ecc-15. diptericin transcripts were apparent $3 \mathrm{~h}$ after infection, and maximal levels were detected in the pupal stage; diptericin expression was not detectable in adults (Fig. $1 B$ ).
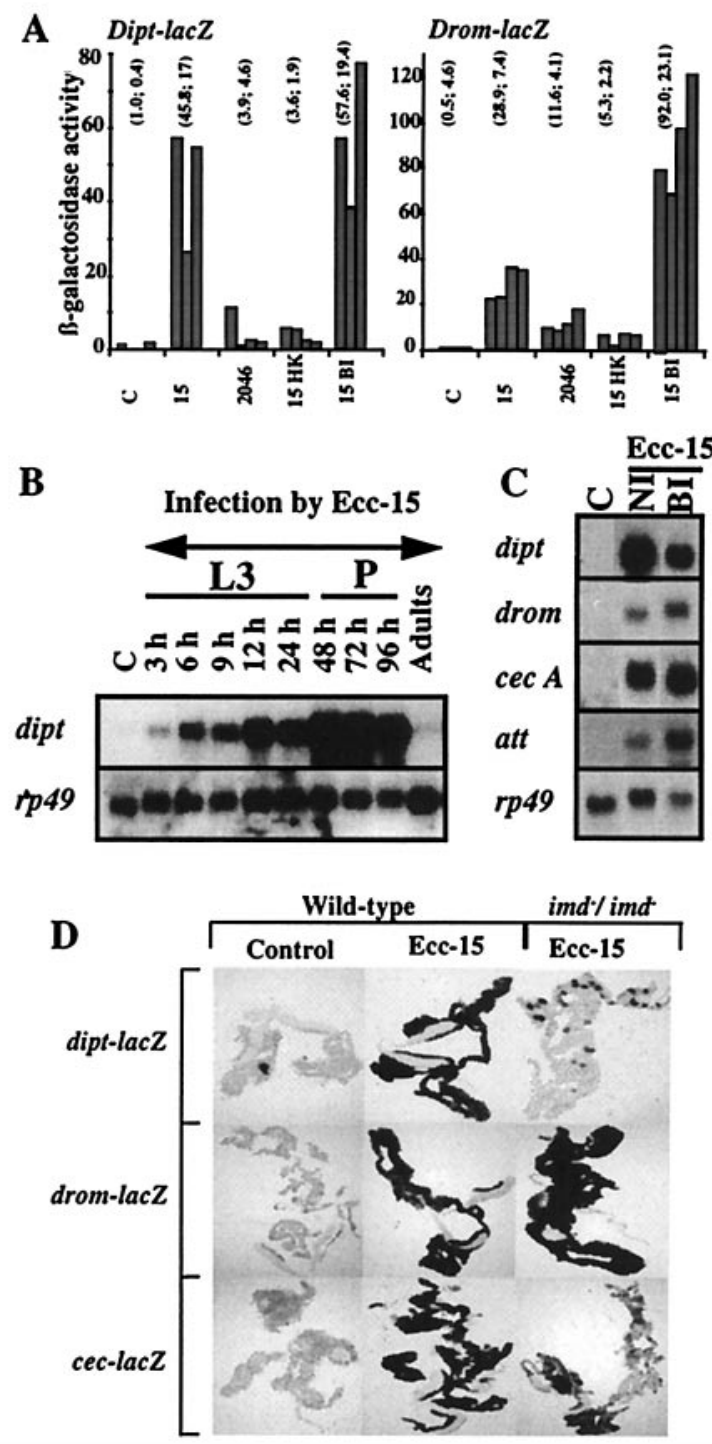

Fig. 1. Induction of antimicrobial peptide genes after Ecc-15 infection. ( $A$ ) Viable Ecc-15 is required to induce a larval immune response. Quantitative measurements of $\beta$-galactosidase activity in larvae carrying either the dipt-lacZ (Left) or the drom-lacZ (Right) reporter genes were performed on five larvae collected $24 \mathrm{~h}$ after various treatments. Each bar represents an independent measurement; mean and SD are indicated above. C, control larvae incubated in banana alone; 15 and 2046, larvae naturally infected with Ecc-15 and Ecc-2046, respectively; 15-HK, heat-inactivated Ecc-15 (40 $\min$ at $\left.70^{\circ} \mathrm{C}\right) ; 15-\mathrm{BI}$, larvae injected with Ecc-15 (bacterial injection). (B) Northern blot analysis shows that Ecc-15 infection induces sustained diptericin expression in larvae and pupae collected at different time intervals after natural infection by Ecc-15. L3, third instar larvae; P, pupae; C, control. (C) Northern blot analysis shows that Ecc-15 infection of larvae induces the expression of all of the antimicrobial genes. Total RNA was collected $24 \mathrm{~h}$ after infection, and the blot was hybridized successively with the following cDNA probes: diptericin (dipt), cecropin A1 (cec A), attacin (att), drosomycin (drom), and ribosomal protein 49 (rp49) as an internal control. C, control larvae incubated with only banana; NI, larvae incubated with Ecc-15 (natural infection); BI, third instar larvae injected with Ecc-15 (bacterial injection). (D) Ecc-15 induces a systemic immune response. Histochemical staining of $\beta$-galactosidase activity is shown in the fat body of wild-type $\left(O r^{R}\right)$ and $i m d$ homozygous larvae that were collected $24 \mathrm{~h}$ after infection and that carry the dipt-lacZ, cec-lacZ, or drom-lacZ reporter genes. Ecc-15 infection induces high expression of all three reporter genes in fat bodies from wild-type larvae. The absence of $\beta$-galactosidase activity in fat bodies isolated from infected imd larvae carrying the dipt-lac $Z$ and cec-lac $Z$ genes indicates that the induction of cec-lac $Z$ and dipt-lac $Z$ expression by Ecc-15 requires the imd gene product. 


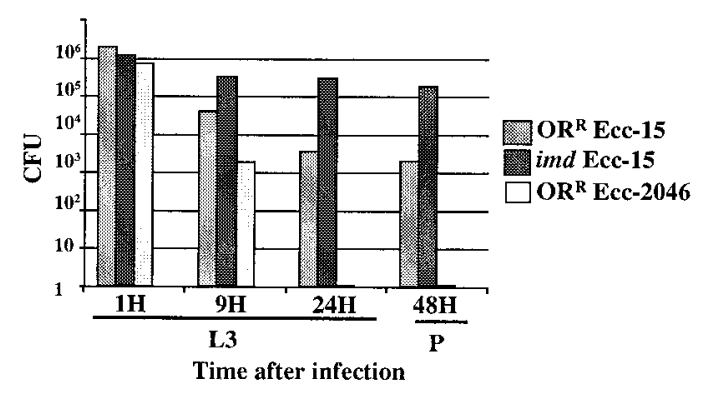

Fig. 2. Ecc-15 persists in developing larvae and pupae. Bacterial persistence was measured in wild-type $\left(\mathrm{Or}^{\mathrm{R}}\right)$ and $i m d / i m d$ mutant lines by plating appropriate dilutions of homogenates of five surface-sterilized larvae or pupae that were naturally infected with rifampicin-resistant strains of Ecc-15 (Ecc-15 rif $\left.{ }^{R}\right)$ and Ecc-2046 (Ecc-2046 rifR) and collected at different times after infection. Bacterial counts were obtained by plating the larval and pupal homogenates on LB medium containing rifampicin $(100 \mu \mathrm{g} / \mathrm{ml}$; ref. 14). The number of colony-forming units (cfu) per larva obtained at each time point after infection represents the mean of three independent measurements. L3, third instar larvae; P, pupae.

diptericin expression in the pupal stage was not caused by reinfection by bacteria from the medium, because larvae that were washed and briefly sterilized $9 \mathrm{~h}$ after infection still expressed high levels of diptericin as pupae (data not shown).

Direct injection of many microorganisms into the body cavity of larvae or adults induces the transcription of all genes encoding antibacterial and antifungal peptides. In contrast, in both larvae and adults, natural infections by entomopathogenic fungi selectively induce the expression of the antifungal genes drosomycin and metchnikowin via the Toll pathway $(3,8)$. The identification of infectious Erwinia strains enabled us to determine whether bacterial infection also leads to an adapted antibacterial response in larvae. Surprisingly, Northern blot analysis shows that natural Ecc-15 infection as well as direct injection into the larval body cavity induces the expression of all of the genes encoding antimicrobial peptides that we analyzed, including the antifungal peptide gene drosomycin (Fig. $1 C$ for cecropin A, attacin, and drosomycin; data not shown for the others). Quantitative measurements of drosomycin gene expression with a drom-lacZ reporter gene indicate, however, that the drosomycin gene is expressed at significantly lower levels in larvae naturally infected by Ecc-15. $\beta$-Galactosidase activity in larvae carrying drom-lacZ is $\approx 3.1$ times higher after directly injecting Ecc-15 than after natural infection. In dipt-lac $Z$ larvae, $\beta$-galactosidase activity is only 1.2 times higher after direct injection of Ecc-15 (Fig. 1A). These expression patterns indicate that natural bacterial infections of Drosophila induce a global antimicrobial response, probably by activating multiple defense-related signaling pathways; however, the antibacterial peptide gene diptericin is induced more strongly than the antifungal peptide gene drosomycin.

The larval fat body, the functional equivalent of the mammalian liver, is located inside the larval hemocoel and is the major site of expression of antimicrobial peptides (1). Natural Ecc-15 infections induce the expression of antibacterial and antifungal peptide reporter genes in wild-type larval fat body cells, indicating that Ecc-15 activates a systemic immune response (Fig. $1 D)$. dipt-lac $Z$ and cec-lac $Z$ but not drom-lac $Z$ expression required the imd gene product; this observation was corroborated by Northern blot analysis (data not shown) to confirm the role of the imd pathway in regulating the activity of the genes encoding antibacterial peptides (13). In addition to a systemic immune response, natural Ecc-15 infections also induce local immune responses (20) that we will describe elsewhere.
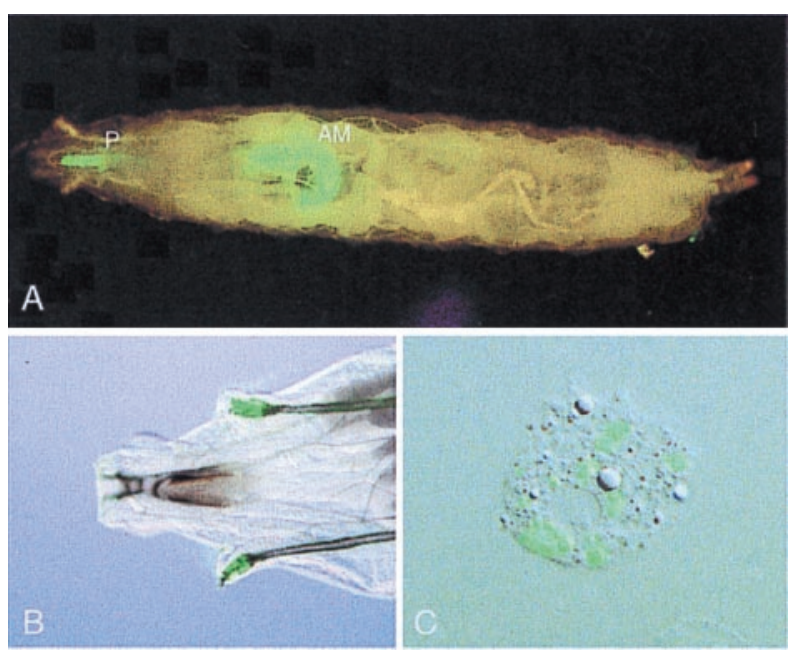

Fig. 3. Fate of Ecc-15 bacteria in the larvae. The localization of Ecc-15 within larvae was determined by using a strain of Ecc-15 (Ecc-15-GFP) that expresses the GFP reporter gene. $(A)$ In a larva naturally infected by Ecc-15-GFP, bacteria are clearly present in the pharynx and anterior midgut. $(B)$ In a larva infected by Ecc-15-GFP, bacteria are present in the anterior spiracles. (C) Hemolymph collected from larvae infected for $16 \mathrm{~h}$ with Ecc-15 shows GFP-marked bacteria phagocytosed by hemocytes. P, pharynx; AM, anterior midgut.

Ecc-15 Persistence and Localization in Infected Larvae. To begin to determine how Ecc-15 infects Drosophila larvae and induces a systemic immune response, we examined Ecc-15 persistence and localization in infected larvae. We first compared Ecc-15 and Ecc-2046 persistence by quantifying bacterial levels in larvae at different time points after natural infection. Both Ecc-15 and Ecc-2046 levels decreased rapidly with time; however, Ecc-15 levels remained consistently higher than those of Ecc-2046, indicating specific Ecc-15 adaptations for survival inside Drosophila (Fig. 2). Ecc-15 persistence could explain the increase in antimicrobial gene expression that we observed at the pupal stage, because Ecc-15 cells may be released into the larval hemolymph during the histolysis of larval tissue that occurs during metamorphosis (Fig. $1 B$ ). Interestingly, Ecc-15 persists at higher levels in imd larvae than in wild-type larvae, suggesting that antibacterial peptides play a significant role in reducing bacterial levels (Fig. 2). Bacteria were rarely detected in adult flies derived from naturally infected wild-type and imd larvae, indicating that Ecc-15 is poorly transmitted to adults.

We studied Ecc-15 localization in larvae naturally infected with an Ecc-15 strain carrying a constitutively expressed GFP reporter gene on a plasmid (15). After 5-7 h of infection, fluorescing bacteria were predominantly present in the digestive tract, most frequently in the foregut and anterior midgut (Fig. $3 A$ ). Although, we also observed bacteria in the respiratory system, especially in the anterior spiracles (Fig. $3 B$ ), the digestive tract appears to be the main route for Ecc-15 infection, because dipt-lac $Z$ larvae sealed at the mouth by ligaturing with a strand of human hair before Ecc-15 exposure contained less $\beta$-galactosidase activity than unsealed larvae (Fig. 4). Bacteria in the digestive tract persisted for several hours, and $16 \mathrm{~h}$ after infection, 5\% of larvae still contained high levels of GFP in the gut. To determine whether Ecc-15 crosses the digestive tract and enters the body cavity, we screened for bacteria expressing GFP in hemolymph collected from larvae $16 \mathrm{~h}$ after infection by Ecc-15-GFP. The results were variable: Ecc-15 was not detected in the hemolymph from most larvae, although larvae that showed high levels of GFP in the gut sometimes contained both motile and stationary bacteria in the hemolymph. Occasionally these bacteria were aggregated with or phagocytosed by hemo- 


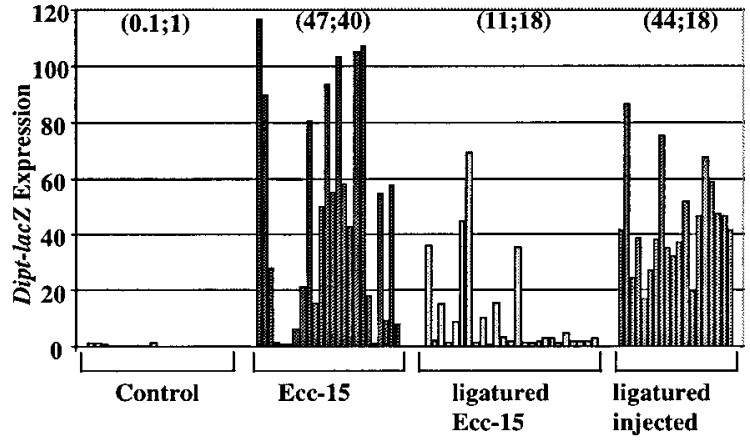

Fig. 4. Ecc-15 infects larvae through the digestive tract. dipt-lacz larvae that were sealed at the mouth by ligaturing with a strand of human hair were naturally infected by Ecc-15 for $12 \mathrm{~h}$. Each bar represents the level of $\beta$-galactosidase activity measured in one larva. Means and SD are indicated above. Ligatured larvae generally failed to express dipt-lacZ after exposure to Ecc-15, indicating that the digestive tract is the major route of infection by Ecc-15. Ligatured larvae still have an wild-type immune response after Ecc-15 injection, indicating that the ligature blocks only the route of infection and does not alter host defense response. Control, untreated larvae; Ecc-15, larvae infected by Ecc-15; ligatured Ecc-15, ligatured larvae infected by Ecc-15; ligatured injected, ligatured larvae collected $8 \mathrm{~h}$ after Ecc-15 injection.

cytes (Fig. 3C). The latter observations suggest that, as observed with protozoan parasites such as Plasmodium that infect mosquitoes (21) and with many mammalian enteric pathogens (22), Ecc-15 can cross larval gut tissues and enter the hemocoel. We believe that most bacteria crossing the gut do not persist in the hemolymph, because we observed bacteria in only a few larvae that were heavily infected in the gut. The crossing of the digestive tract may explain how Ecc-15 triggers a systemic immune response, although we cannot rule out the possibility that the presence of bacteria inside the hemocoel is only a consequence of severe infection and not the cause of antimicrobial peptide synthesis.

Evidence That Hemocytes Are Required to Activate Antibacterial Gene Expression After Ecc-15 Infection. dom and l(3)hem are two cell proliferation mutations that affect diploid structures of the larvae (i.e., neuroblasts, imaginal discs, germ cells, and the lymph gland) and greatly reduce hemocyte number $(8,9)$. To determine whether circulating hemocytes play a role in the immune response induced by Ecc-15, we constructed strains carrying either the dom or l(3)hem mutations in combination with dipt-lacZ and drom-lacZ. Strikingly, both the dom and l(3)hem mutations decreased dipt-lac $Z$ expression in the fat body after Ecc-15 natural infection but not after direct injection of Ecc-15 (Fig. $5 A$ and $B$ for l(3)hem; data not shown for dom). These results confirm previous reports showing that immune responses after bacterial injection remain inducible in dom and $l(3)$ hem mutant larvae $(8,9)$. The effect of the dom and $l(3)$ hem mutations on the Ecc-15-mediated induction of dipt-lacZ after natural infection, however, suggests that hemocytes play a more significant role in activating a systemic antibacterial response in the absence of physical injury. Our hypothesis is that the presence of bacteria inside the hemocoel is not, by itself, a sufficient stimulus to trigger a systemic response; induction of the systemic responses requires a second signal either from hemocytes or from physical injury. This requirement would explain why dom larvae, which often contain bacteria in their hemocoel, do not express the diptericin gene (8). It is possible that cytokine-like molecules emanating from hemocytes in contact with bacteria can signal to the fat body. Alternatively, a product synthesized by hemocytes and secreted into the hemolymph, for example a receptor for Gram-negative bacteria, could
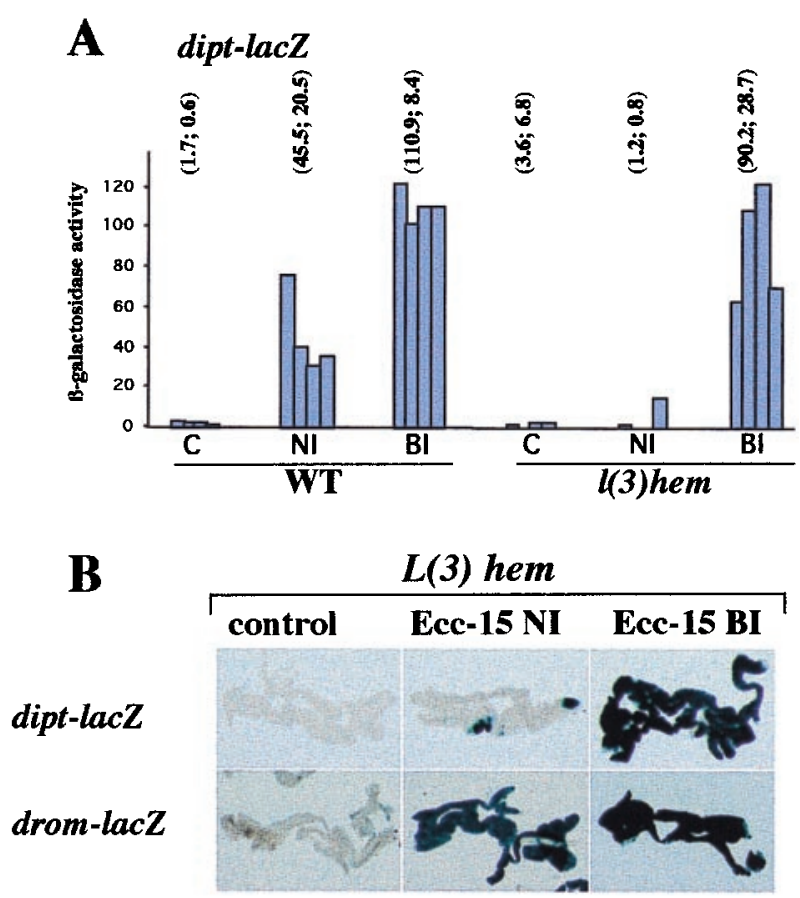

Fig. 5. diptericin induction after natural infection by Ecc-15 requires hemocytes. (A) Quantitative measurements of $\beta$-galactosidase activity performed on five larvae collected $24 \mathrm{~h}$ after natural infection or bacterial injection by Ecc-15. Experiments were performed as described (3) on larvae carrying the dipt-lacZ reporter gene in either wild-type (WT) or I(3)hem/I(3)hem mutant backgrounds. C, control; NI, larvae incubated with Ecc-15 (natural infection); $\mathrm{BI}$, third instar larvae injected with Ecc-15 (bacterial injection). Each bar represents an independent measurement. Means and SD are indicated above. $(B)$ Histochemical staining of $\beta$-galactosidase activity is shown in the fat body of I(3)hem homozygous larvae carrying either the dipt-lacZ or drom-lacZ reporter genes. In contrast to injection, natural Ecc-15 infection failed to induce a strong expression of the dipt-lacZ reporter gene in the I(3)hem fat body. Larval fat bodies were isolated $24 \mathrm{~h}$ after infection and stained (5bromo-4-chloro-3-indolyl $\beta$-D-galactoside) as described (11). C, unchallenged control larvae; Ecc-15 NI, natural infection by Ecc-15; Ecc-15 BI, bacterial injection of Ecc-15.

mediate antibacterial responses. Bacterial injection seems to trigger additional pathways that bypass hemocytes to regulate immune responses in the fat body. We cannot, however, exclude that $d o m$ and $l(3)$ hem mutations alter other physiological process that are necessary for Ecc-15-mediated antibacterial gene expression. Finally, drom-lac $Z$ remains at least partially inducible after Ecc-15 infection in dom and l(3)hem mutants (Fig. 5); these data are consistent with the regulation of this antifungal peptide gene by signaling pathways that are largely distinct from those that regulate the antibacterial genes. This result is also consistent with the observation of Braun et al. (8) showing that natural infection by entomopathogenic fungi induces the expression of the drosomycin gene in dom larvae.

\section{Conclusions}

Our results identify bacterial species that induce Drosophila immune responses in the absence of physical injury. Because we use high concentrations of these Erwinia strains to induce high levels of antimicrobial gene expression consistently, we cannot conclude that similar levels of induction are achieved in natural habitats. We do argue, however, that the infectious Erwinia strains possess specific, unique mechanisms for infecting Drosophila and that these infections can induce antimicrobial gene expression. This conclusion is supported both by previous studies that identify interactions between Drosophila and Erwinia spe- 
cies $(6,7)$ and by our observation that, even at similarly high concentrations, no other bacterial species tested induces a similar immune response. The identification of bacterial species that naturally infect Drosophila provides a powerful tool for studying the mechanisms and signaling pathways that regulate Drosophila immune responses. We show that Erwinia infections activate a partially adapted immune response by inducing the expression of genes encoding antibacterial peptides to a higher level than that of genes encoding antifungal peptides. In addition, our data suggest that hemocytes play a role in inducing these antibacterial responses in the fat body after natural infection through the digestive tract; future challenges include identifying the additional factors that mediate this response. Studies of the interplay between bacterial pathogens and their plant and animal hosts illustrate the complexity of host-pathogen interactions (22). Recently, the plant species Arabidopsis thaliana and the nematode Caenorhabditis elegans, both amenable to genetic manipulation, were developed as model systems for studying pathogenesis by the broad host range, opportunistic bacterial pathogen Pseudomonas aeruginosa $(23,24)$. The identification of a bacterial species amenable to genetic analysis that specifically infects Drosophila provides an opportunity to dissect genetically a very specific host-pathogen interaction as well as the regula-

1. Hoffmann, J. A. \& Reichhart, J.-M. (1997) Trends Cell Biol. 7, 309-316.

2. Hoffmann, J. A., Kafatos, F. C., Janeway, C. A. \& Ezekowitz, R. A. B. (1999) Science 284, 1313-1318.

3. Lemaitre, B., Reichhart, J. M. \& Hoffmann, J. A. (1997) Proc. Natl. Acad. Sci. USA 94, 14614-14619.

4. Agrios, G. N. (1997) Plant Pathology (Academic, New York), 4th Ed

5. Pérombelon, M. C. M. (1980) Annu. Rev. Phytopathol. 18, 361-387.

6. Molina, J. J., Harisson, M. D. \& Brewer, J. W. (1974) Am. Potato J. 51, 245-250.

7. Kloepper, J. W., Brewer, J. W. \& Harrison, M. D. (1981) Am. Potato J. 58, $165-175$.

8. Braun, A., Hoffmann J. A. \& Meister M. (1998) Proc. Natl. Acad. Sci. USA 95, 14337-14342.

9. Gateff, E. (1994) Ann. N.Y. Acad. Sci. 712, 260-279.

10. Reichhart, J. M., Meister, M., Dimarcq, J. L., Zachary, D., Hoffmann, D., Ruiz, C., Richards, G. \& Hoffmann, J. A. (1992) EMBO J. 11, 1469-1477.

11. Manfruelli, P., Reichhart, J. M., Steward, R., Hoffmann J. A. \& Lemaitre, B. (1999) EMBO J. 18, 3380-3391.

12. Engström, Y., Kadalayil, L., Sun, S. C., Samakovlis, C., Hultmark, D. \& Faye, I. (1993) J. Mol. Biol. 232, 327-333.

13. Lemaitre, B., Kromer-Metzger, E., Michaut, L., Nicolas, E., Meister, M., tion of host immune responses. The sharing of infectious strategies between a wide range of prokaryotic pathogens and the conservation of multiple aspects of innate immunity suggest that our analysis of Drosophila-Erwinia interactions will have a global relevance. Finally, insects have been recognized as vectors for many plant and human pathogenic bacteria for a long time; our study of the Drosophila-Erwinia interaction shows that insects are not passive carriers of these microorganisms, but, as observed for parasites, complex interactions can occur between the two partners.

We thank Philippe Sansonetti and Michelle Rathman (Institut Pasteur, Paris) for providing plasmid pFPV25.1 and George Salmond (Cambridge University, Cambridge, U.K.) and Arun Chatterjee (University of Missouri, Columbia, MO) for sending Erwinia Strains. We thank our colleagues Cecile Vialet, Ariane Gratias, Cynthia Alland, François Leulier, Marie Meister, Jean Luc Imler, and Dominique Expert for stimulating discussions. The technical assistance of Reine Klock, Raymonde Syllas, and Martine Schneider is gratefully acknowledged. This work was supported by the Centre National de la Recherche Scientifique, the Fondation pour la Recherche Médicale, and Program Microbiologie Grant PRMMIP98. R.S.K. is supported by the Fondation pour la Recherche Médicale.

Georgel, P., Reichhart, J. M. \& Hoffmann, J. A. (1995) Proc. Natl. Acad. Sci. USA 92, 9465-9469.

14. Miller, J. H. (1972) Experiments in Molecular Genetics (Cold Spring Harbor Lab. Press, Plainview, NY).

15. Valdivia, R. H. \& Falkow S. (1996) Mol. Microbiol. 22, 367-378.

16. Åsling, B., Dushay, M. S. \& Hultmark, D. (1995) Insect Biochem. Mol. Biol. 25, 511-518.

17. Tryselius, Y., Samakovlis, C., Kimbrell, D. A. \& Hultmark, D. (1992) Eur. J. Biochem. 204, 395-399.

18. Fehlbaum, P., Bulet, P., Michaut, L., Lagueux, M., Broekaert, W. F., Hetru, C. \& Hoffmann, J. A. (1994) J. Biol. Chem. 269, 33159-33163.

19. O'Connel, P. \& Rosbach, M. (1984) Nucleic Acids Res. 12, 5495-5513.

20. Ferrandon, D., Jung, A. C., Criqui, M. C., Lemaitre, B., Uttenweiler-Joseph, S., Michaut, L., Reichhart, J. M. \& Hoffmann, J. A. (1998) EMBO J. 17, 1217-1227.

21. Shahabuddin, M. (1998) Parasitology 116, 83-93.

22. Finlay, B. B. \& Falkow S. (1997) Microbiol. Mol. Rev. 61, 136-169.

23. Rahme, L. G., Tan, M. W., Le, L., Wong, S. M., Tompkins, R. G., Calderwood, S. B. \& Ausubel, F. M. (1997) Proc. Natl. Acad. Sci. USA 94, 13245-13250.

24. Mahajan-Miklos, S., Tan, M. W., Rahme, L. G. \& Ausubel, F. M. (1999) Cell 96, 47-56.

25. Flyg, C., Kenne, K. \& Boman, H. G. (1980) J. Gen. Microbiol. 120, 173-181. 